EXTENDED REPORT

\title{
FTY720 prolongs clear corneal allograft survival with a differential effect on different lymphocyte populations
}

\author{
K Mayer, F Birnbaum, T Reinhard, A Reis, S Braunstein, F Claas, R Sundmacher
}

Br J Ophthalmol 2004;88:915-919. doi: 10.1136/bjo.2003.034017

\author{
See end of article for \\ authors' affiliations \\ Correspondence to: \\ Dr K Mayer, Eye Hospital, \\ Heinrich-Heine University, \\ Moorenstr 5, 40225 \\ Düsseldorf, Germany; \\ Klaus.mayer@ \\ uni-duesseldorf.de \\ Accepted \\ 30 November 2003
}

\begin{abstract}
Background: FTY720 is a potent immunomodulator with unique effects on lymphocyte homing and has recently proved to be safe and effective in renal transplantation in man. The authors investigated the potency of FTY720 in inhibiting allograft rejection in the rat model of orthotopic allogeneic penetrating keratoplasty.

Methods: Penetrating keratoplasties were performed using Fisher rats as donors and Lewis rats as recipients or donors: group $1(n=10)$, allogeneic control; group $2(n=10)$, Lewis/Lewis syngeneic control; group 3 ( $\mathrm{n}=9$ ), mycophenolate mofetile (MMF) $40 \mathrm{mg} / \mathrm{kg}$; group 4 ( $\mathrm{n}=10)$, FTY720 $1.2 \mathrm{mg} / \mathrm{kg}$; group 5 $(\mathrm{n}=8)$, FTY720 $0.3 \mathrm{mg} / \mathrm{kg}$. Four animals from each group were sacrificed for immunohistological evaluation on day 14. Medication in the therapy groups was given for 18 days.

Results: The mean (SD) rejection free graft survival time was $11.3(0.8)$ days for the allogeneic control (group 1), 24.6 (2.5) days for group 3 (MMF), 44.5 (5.7) days for group 4 (FTY720 $1.2 \mathrm{mg} / \mathrm{kg}$ ), and 35.3 (5.7) days for group 5 (FTY720 $0.3 \mathrm{mg} / \mathrm{kg})(\mathrm{p}<0.05)$. The allogeneic control showed a dense infiltration with CD4+, CD8+, CD161+ (NK-cells), CD25+ (IL2 receptor), and macrophages. In the therapy groups the density of infiltrating CD4+, CD8+, CD161+ (NK-cells), and CD25+ (IL2 receptor) cells was notably reduced compared with the allogeneic control $(p<0.05)$. In group 5 however, the reduction of infiltration by CD4+ cells was higher than the reduction of infiltration by $C D 8+(p<0.05)$ and $C D 161+$ (NK) cells.

Discussion: Oral immunosuppression with FTY720 significantly prolongs corneal allograft survival in this transplant model. The results suggest that FTY720 has a different effect on certain lymphocyte populations. CD4+ cells seem to be more affected than CD8+ cells and NK-cells.
\end{abstract}

$\mathrm{T}$ he novel immunomodulator FTY720 is a synthetic structural analogue of myriocin, a metabolite of the ascomycete Isaria sinclairii. ${ }^{1}$ It has a remarkable potency in inhibiting rejection of allografts in various experimental animal models ${ }^{2-7}$ and has recently proved to be safe and effective in renal transplantation in man. ${ }^{8-10}$ The protective effect correlates with a sequestration of lymphocytes from the peripheral circulation to lymph nodes and Peyer's patches, leading to a reduced infiltration into inflammatory sites and grafted organs. Unlike the currently used immunosuppressive agents FTY720 does not inhibit $\mathrm{T}$ cell activation and proliferation and in rodent models does not impair immunity to systemic viral infection. ${ }^{3411}{ }^{12}$ It could be shown that an enhanced lymphocyte migratory response to chemokines may be a main cause of FTY720 induced lymphocyte sequestration. ${ }^{13}{ }^{14}$ Recent results suggest that FTY720, after phosphorylation, acts through sphingosine 1-phosphate signalling pathways to modulate chemotactic responses and lymphocyte trafficking. ${ }^{15} 16$

We investigated the potency of FTY720 in inhibiting allograft rejection in the rat model of orthotopic allogeneic penetrating keratoplasty.

\section{MATERIAL AND METHODS \\ Animals}

Forty seven allogeneic orthotopic penetrating keratoplasties were performed using Fisher rats $\left(\mathrm{RT}^{\mathrm{lvl}}\right)$ as donors (in the allogeneic groups) and Lewis rats $\left(\mathrm{RTl}^{1}\right)$ as recipients (in all study groups) or donors (in the syngeneic group). All animals were females, weighing 180-220 g. The animals were obtained and cared for in accordance with the Directives of the European Community as well as with the recommendations of the National Institutes of Health's Guide for the Care and Use of Laboratory Animals, NIH Publication Number 8523 (revised 1985).

\section{Operative technique}

We performed an orthotopic penetrating keratoplasty according to the technique of Herbort et al. ${ }^{17}$ Mydriasis in the eyes of both the donors and the recipients was achieved by local application of phenylephrine-HCL $10 \%$ eyedrops (Neosynephrin-POS, Ursapharm). These eyedrops were administered three times at intervals of 10 minutes before the operation. Thereafter, the donors were killed and corneal grafts were obtained using a $3.5 \mathrm{~mm}$ trephine. Until implantation, donor buttons were stored at room temperature for approximately 20 minutes in a conservation medium for corneas (Biochrom, Berlin, Germany).

Recipients, after a brief inhalation anaesthesia with Halothan (Hoechst-Roussel, Bad Soden, Germany), were anaesthetised with an intraperitoneal mixed injection of ketamine hydrochloride (Ketanest 50, Parke-Davis, Pfizer, New York, NY, USA) $60 \mathrm{mg} / \mathrm{kg}$ bodyweight, xylazin (Rompun $2 \%$, Bayer, Leverkusen, Germany) $3 \mathrm{mg} / \mathrm{kg}$ bodyweight, and atropine sulfate $0.1 \mathrm{mg}$ (Atropinsulfat, B Braun, Melsungen, Germany), and fixed in a dextral lateral position. After the left recipient cornea had been removed with a $3.0 \mathrm{~mm}$ trephine, the donor cornea was transplanted. The transplant was sewn in with eight interrupted sutures (Ethicon 11.0, Ethicon, Somerville, NJ, USA) which were subsequently left in place to enhance neovascularisation and thus accelerate the rejection process. The anterior chamber of the eye was restored at the end of the operation by injection of balanced

Abbreviations: BSS, balanced salt solution; MMF, mycophenolate mofetil. 
salt solution (BSS). To protect the transplant, a blepharorraphy was performed with two interrupted sutures (Prolene 6.0, Ethicon) which remained in place for three days, and gentamicin (Refobacin, Merck, Darmstadt, Germany) ointment was applied.

\section{Groups}

The groups were divided up as follows:

Group $1(\mathrm{n}=10)$ : Fisher/Lewis (allogeneic control)

Group $2(\mathrm{n}=10)$ : Lewis/Lewis (syngeneic control)

Group $3(\mathrm{n}=9)$ : Fisher/Lewis (mycophenolate mofetil (MMF) $40 \mathrm{mg} / \mathrm{kg}$ bodyweight/day)

Group $4(\mathrm{n}=10)$ : Fisher/Lewis (FTY720 $1.2 \mathrm{mg} / \mathrm{kg}$ bodyweight/day)

Group $5(\mathrm{n}=8)$ : Fisher/Lewis (FTY720 $0.3 \mathrm{mg} / \mathrm{kg}$ bodyweight/day).

Medication in the therapy groups was given orally with a stomach tube, starting on the day of operation and continuing daily for 18 days. FTY720 was dissolved in water $(0.4 \mathrm{mg} / \mathrm{ml})$. MMF was given as a suspension in water (15 mg/ml).

\section{Clinical evaluation}

Twenty seven rats (group $1(n=6)$, group $2(n=6)$, group 3 $(n=5)$, group $4(n=6)$, group $5(n=4))$ were subjected to clinical examination for a maximum of 100 days. Each animal was examined by slit lamp microscopy during a brief inhalation anaesthesia with Halothan (Hoechst-Roussel).

The transplants were evaluated every third day by means of a scoring system which assessed opacity, oedema, and neovascularisation. The main criterion was opacification of the graft. To enhance accuracy of graft evaluation, each animal was evaluated by two examiners (KM and FB). The observers were not masked for treatment condition.

Opacity: $0=$ no opacity; 1 = slight opacity (details of iris clearly visible); $2=$ some details of iris no longer visible; $3=$ pronounced opacity (pupil still recognisable); $4=$ total opacity.

After 100 days, or following clinically diagnosed complete opacification of the transplant, the recipient animals were sacrificed through the inhalation of $\mathrm{CO}_{2}$. After that, the operated eye was enucleated and fixed in a buffered formalin solution (4\%). For histological assessment the formalin fixed eyes were cut into $7 \mu \mathrm{m}$ thick preparations and subjected to haematoxylin eosin staining.

Additionally, all experimental animals were closely monitored for signs of toxic side effects (for example, weight loss, emaciation) during the whole follow up.

\section{Immunohistology}

In a second set of experiments 20 animals were transplanted and treated according to the aforementioned groups (four from each group). All rats were sacrificed for immunohistological evaluation on day 14 .

Using a cryostat, $7 \mu \mathrm{m}$ thick sections of each frozen eye were prepared. Specimens were allowed to dry overnight on silanised slides, then they were fixed in $100 \%$ aceton at $-20^{\circ} \mathrm{C}$ for 10 minutes. After the aceton had evaporated (30 seconds) the slides were immersed in Tris buffer. The primary antibodies were applied to the sections for one hour at room temperature. Monoclonal mouse antirat antibodies to T helper cells (CD4, W3/25, 1:200), cytotoxic T cells (CD8, MRC OX-8, 1:100), NK cells (CD161, 10/78, 1:200), IL2 receptor (CD25, MRC OX-39, 1:600), macrophages (ED2, 1:300), and B cells (RLN-9D3, 1:100) were used. All monoclonal antibodies were purchased from Serotec, Oxford, UK. The primary antibodies were detected by
DAKO EnVision-HRP (Goat antimouse, DAKO Code No K5007) for 30 minutes at room temperature. Ten per cent rat serum had been added to the EnVision reagent to prevent binding to endogenous rat Ig. Chromogen AEC (DAKO Code No K3461) was applied for $2 \times 5$ minutes to the tissue, followed by counterstaining with DAKO Haematoxilin (DAKO Code No S2020) for 20 seconds. A photograph was taken of every slide. To ensure consistency in evaluating the slides, the photographs were always taken in the same anatomic area-that is, the central zone of each graft. Cells were counted on the photographs to quantify the degree of graft infiltration by each cell type. The photographs were evaluated by a masked examiner (the cell count of one photograph of one high power field per animal is shown in figures 3 to 6 ).

\section{Differential blood count}

Blood samples for differential blood counts were taken from the tail vein on day 7, 25, and 70 and from heart blood when animals were killed on day 14 and on day 100.

\section{Statistical analysis}

Time to rejection was calculated as the time to the event from the date of transplant and statistically compared with the log rank test. The densities of infiltrating immune cells were compared statistically using the non-parametric MannWhitney test.

\section{RESULTS}

\section{Clinical evaluation}

All grafts were slightly opacified three days after surgery when the blepharorraphy was opened. The allogeneic grafts without treatment (group 1) remained in that status for some days and then rapidly progressed to complete opacification with mean graft failure after 11.3 (SD 0.8) days (figs 1 and 2 ). Histological assessment of the enucleated formalin fixed eyes confirmed graft rejection as reason of graft failure.

All other grafts (group 2-5) cleared up during the following two weeks and showed nearly no opacity around day 18 (fig 1). The syngeneic grafts (group 2) then remained clear for the rest of the follow up.

After cessation of therapy rejection occurred in all grafts of all treatment groups (group 3-5) with opacification of the previous clear grafts. An increase of the opacity score by two or more points was defined as time of rejection. In contrast to the allogeneic grafts without therapy none of the grafts in the

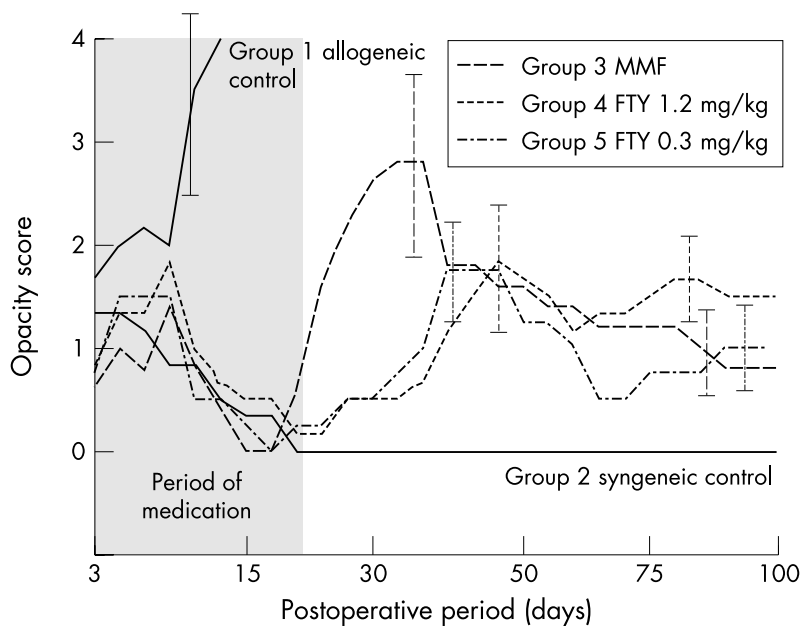

Figure 1 Opacity score of corneal grafts in the control and therapy groups (ranges indicate standard deviation at certain important time points). 


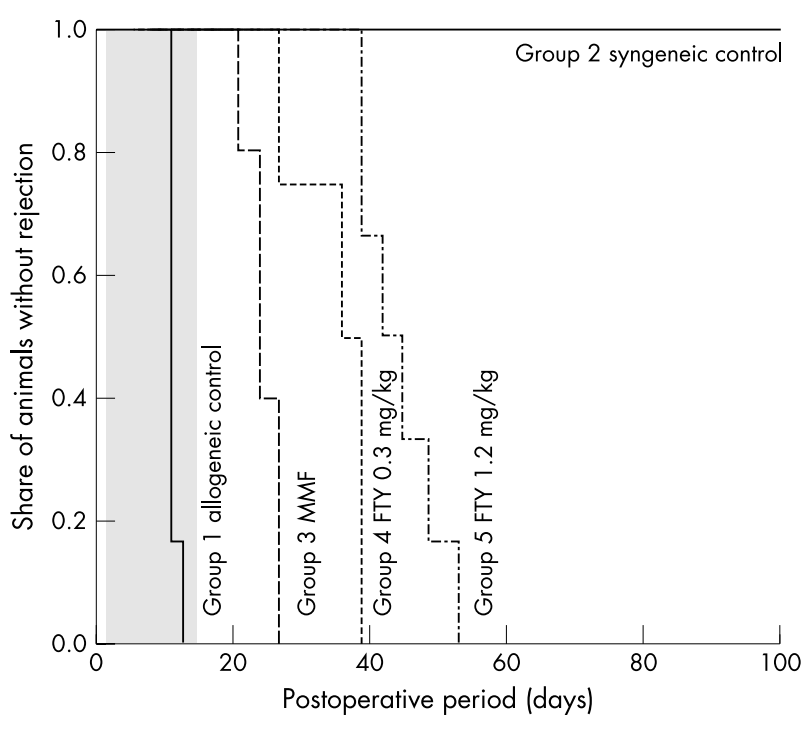

Figure 2 Rejection free graft survival in the control and therapy groups ( $p<0.01$ comparing therapy groups with allogeneic control; $p<0.05$ comparing therapy groups one with another).

treatment groups showed complete opacification during the whole follow up (fig 1). The mean rejection free graft survival time was 24.6 (SD 2.5) days for the group treated with MMF $40 \mathrm{mg} / \mathrm{kg}$ (group 3), 44.5 (SD 5.7) days for the group treated with FTY720 $1.2 \mathrm{mg} / \mathrm{kg}$ and 35.3 (SD 5.7) days for the group treated with FTY720 $0.3 \mathrm{mg} / \mathrm{kg}$ (fig 2). As none of the grafts of the treatment groups showed graft failure with complete opacification of the graft, the animals were followed up for 100 days. Many of the rejected grafts cleared up again (fig 1). At the end of follow up after 100 days, histological assessment of the enucleated eyes did not show any signs of rejection in all grafts of the treatment groups.

\section{Immunohistology}

The allogeneic grafts without therapy (group 1) showed a dense infiltration with CD4+, CD8+, CD161+ (NK-cells), CD25+ (IL2 receptor), and ED2+ (macrophages) (figs 3-6). There were no or only few infiltrating CD4+, CD8+, CD161+, $\mathrm{CD} 25+$ in the syngeneic grafts (group 2) (figs 3-6). In group 3 (MMF), group 4 (high dose of FTY $1.2 \mathrm{mg} / \mathrm{kg}$ ), and group 5 (low dose of FTY $0.3 \mathrm{mg} / \mathrm{kg}$ ) the density of infiltrating CD4+, CD8+, CD161+ (NK-cells), and CD25+ (IL2 receptor) cells was notably reduced compared with the allogeneic grafts without therapy (group 1) (figs 3-6). In group 5 however, the reduction of infiltration by CD4+ cells (fig 3) was higher than the reduction of infiltration by CD8+ and CD161+ (NK) cells (figs 4 and 5$)(p<0.05$ comparing the reduction of infiltration by CD4+ cells with the reduction of infiltration by CD8+ cells). In two grafts of group 5 the infiltration with CD161+ cells was relatively high and in the other two relatively low. Due to the high variance of infiltration with CDl61+ cells in group 5 , the difference between reduction of infiltration by CD4+ cells and CD161+ cells is not statistically significant.

No B cells were present in any of the grafts.

\section{Differential blood count}

In group 4 (high dose of FTY $1.2 \mathrm{mg} / \mathrm{kg}$ ) and group 5 (low dose of FTY $0.3 \mathrm{mg} / \mathrm{kg}$ ) we observed reduced percentages of lymphocytes under FTY720 therapy in differential blood counts (fig 7). On day 25, one week after cessation of FTY720, the percentage of lymphocytes was close to normal in group 5. In group 4 lymphocytes were still reduced at that time. On

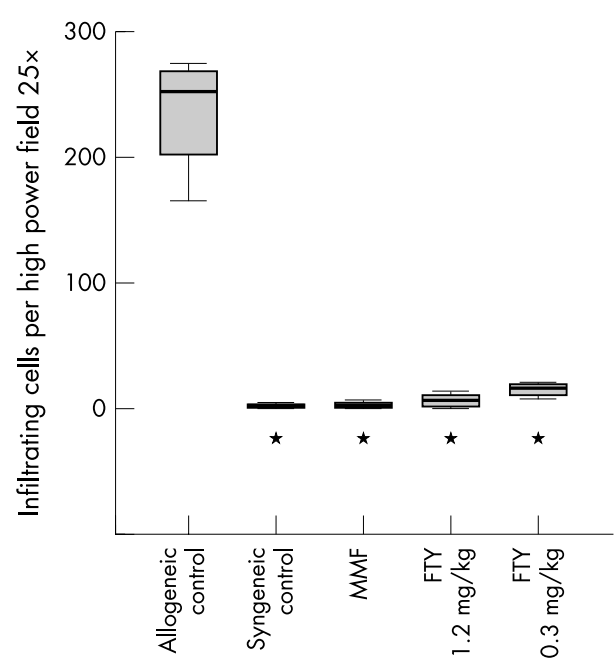

Figure 3 Immunohistology of cornea grafts. Density of infiltrating CD4+ cells. ( ${ }^{*} p<0.05$ compared with allogeneic control).

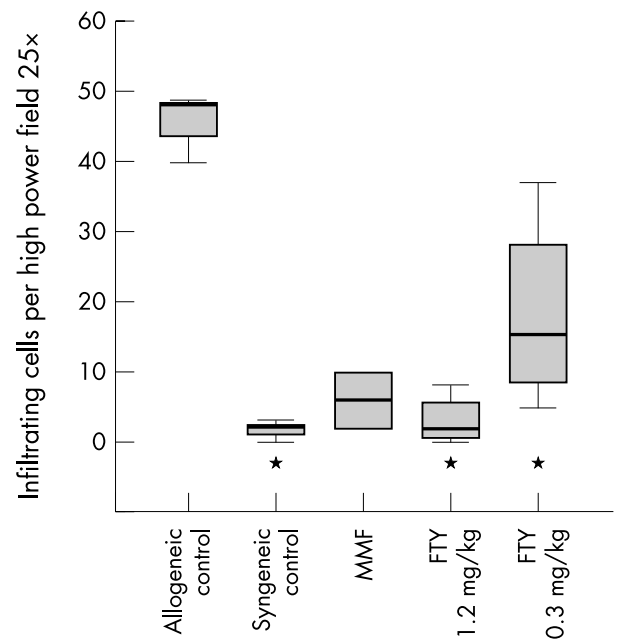

Figure 4 Immunohistology of cornea grafts. Density of infiltrating CD8+ cells. ( ${ }^{*} p<0.05$ compared with allogeneic control).

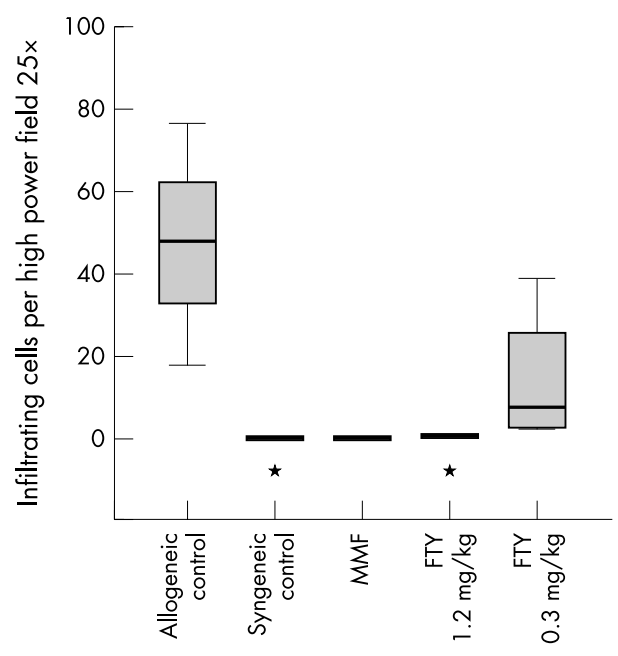

Figure 5 Immunohistology of cornea grafts. Density of infiltrating $\mathrm{CD} 161+$ cells (NK-cells). (" $\mathrm{p}<0.05$ compared with allogeneic control). 


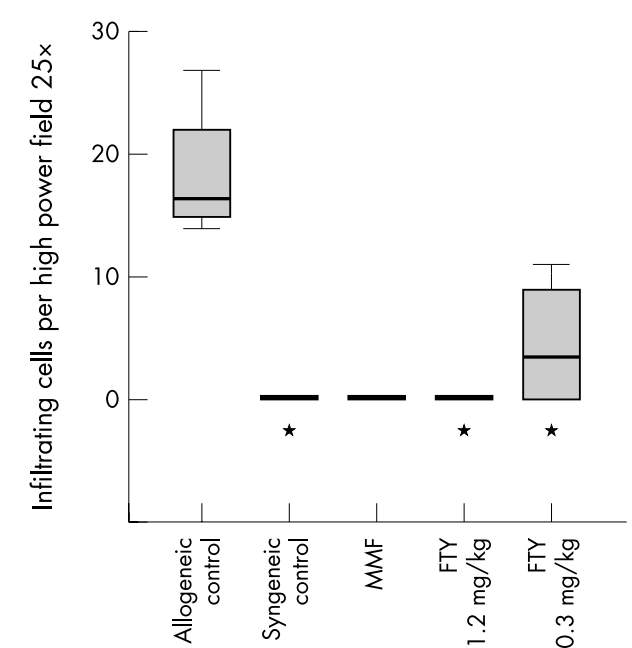

Figure 6 Immunohistology of cornea grafts. Density of infiltrating CD25+ cells (IL2 receptor). ( ${ }^{*} p<0.05$ compared with allogeneic control).

day 70 and on day 100 both FTY720 groups had normal differential blood counts.

We decided to puncture the tail vein and not to burden the animals with tail clipping. Therefore we were only able to make blood smears for differential blood counts and did not get absolute lymphocyte counts, which would reveal further useful information.

\section{Compatibility of FTY720 and MMF}

All experimental animals were closely monitored for signs of toxic side effects (weight loss, emaciation) during the whole follow up. In group 3 (MMF) we observed statistically significant weight loss between day 0 (206 (SD 8) g) and day 18 (188 (SD 12) g) $(\mathrm{p}<0.05$, Student's $t$ test). After cessation of therapy on day 18 these animals were gaining weight again with 220 (SD 12) g on day 100. Animals from all other groups gradually gained weight during follow up. Some animals in group 3 had soft stool under MMF therapy. No other side effects have been observed in the different treatment protocols.

\section{DISCUSSION}

Protective effects of systemic immunosuppression in high risk keratoplasty have been described by several authors. ${ }^{18-22}$ The two substances being used clinically at the moment are cyclosporin A and MMF. This prophylaxis is not sufficient or compatible after all high risk keratoplasties. Several new immunosuppressive agents have been developed in recent years. The aim has been to find new immunosuppressive drugs with fewer adverse effects and enhanced efficacy than those currently used.

FTY720 is a novel, high potency immune modulating agent that is remarkably effective in a variety of autoimmune and transplant models ${ }^{2-7}$ and has recently proved to be effective in renal transplantation in man. ${ }^{8-10}$ The mechanism of action of FTY720 appears to be distinct from that of any other drug approved or being developed for use in organ transplantation. FTY720 apparently sequesters lymphocytes from circulation to secondary lymph tissue compartments ${ }^{3}$ with concomitant reduction of specific effector $\mathrm{T}$ cells recirculating from the lymph nodes to inflamed peripheral tissues ${ }^{11}$ and graft sites. ${ }^{5}$

In this study we have been able to prove the effect of FTY720 in delaying rejection after allogeneic corneal transplantation, which has not been been described in the literature so far. Therapy with FTY720 $(1.2 \mathrm{mg} / \mathrm{kg}$ and $0.3 \mathrm{mg} / \mathrm{kg}$ ) produced a statistically significantly prolonged

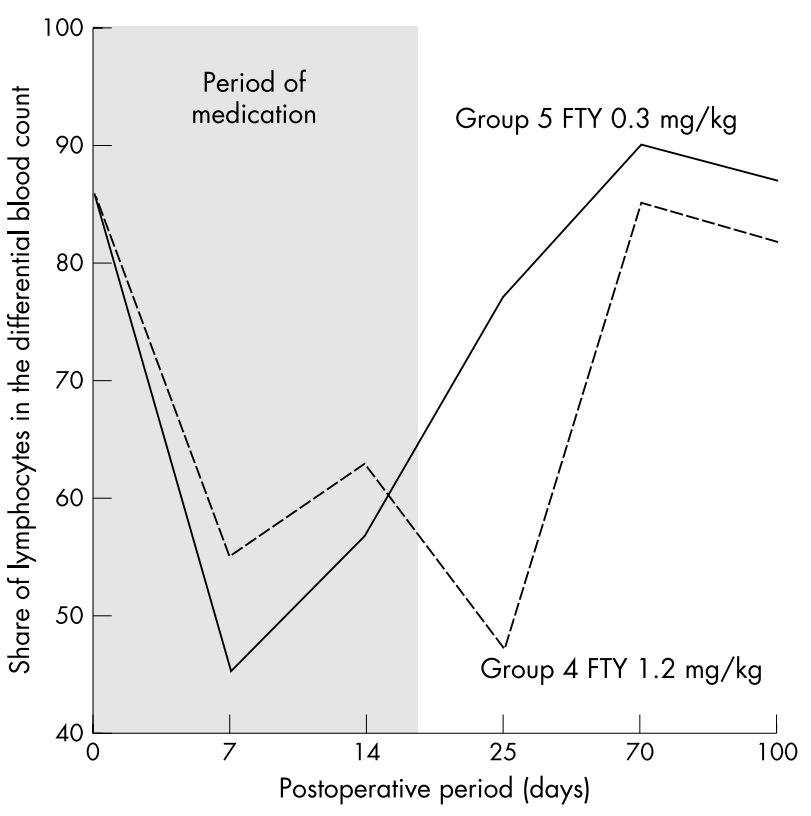

Figure 7 Differential blood counts in the FTY720 therapy groups.

rejection free graft survival $(p<0.01)$ as compared with the allogeneic control (fig 2). We found a statistically significant dose dependent difference between FTY720 $1.2 \mathrm{mg} / \mathrm{kg}$ and $0.3 \mathrm{mg} / \mathrm{kg}(\mathrm{p}<0.05)$.

On day 100 all grafts were clear again and showed no histological signs of rejection. The endothelium of the allogeneic grafts is probably replaced by the endothelium of the host by that time. In contrast to humans, rats seem to be able to regenerate their endothelium. ${ }^{17}$

We found a great reduction of CD4+ cells infiltrating grafts of group 5 (low dose of FTY $0.3 \mathrm{mg} / \mathrm{kg}$ ) like in the other therapy groups, but there was still a considerable number of NK cells and CD8+ cells infiltrating those grafts (figs 3-5). Bold et al and Vaessen et al found in renal transplant patients a stronger reduction of CD4+ cell counts than of CD8+ cell counts in the peripheral blood under FTY720 therapy, whereas NK cells were not affected in those studies. ${ }^{23}{ }^{24}$ These results and our results suggest that FTY720 has a different effect on certain lymphocyte populations. CD4+ cells seem to be affected at most. In group 4 (high dose of FTY $1.2 \mathrm{mg} / \mathrm{kg}$ ) we can see that FTY720 given at higher doses can block the rejection process, and also that NK cells and CD8+ cells can be prevented to a great extent from infiltrating allogeneic corneal grafts (figure 3-6).

The main mediators of corneal graft rejection are CD4+ cells. We also found many NK cells infiltrating the allogeneic grafts without therapy. This confirms previous observations that NK cells might also play a role in corneal graft rejection..$^{25}$ Further studies should evaluate the relative importance of NK cells in corneal graft rejection in relation to CD4+ cells.

Further studies should examine the possible effect on different subtypes of CD4+ cells. For a better understanding of FTY720 in corneal transplantation it would be helpful to look at the distribution pattern of chemokine receptors on CD4+ cells in the blood, lymphoid tissues, and corneal graft. Changes of the distribution pattern of chemokine receptors for inflammatory chemokines (for example, CCR5, CCR3) ${ }^{27}$ and for homeostatic chemokines (for example, CXCR4, CXCR5, and CCR7) ${ }^{27}$ can be expected in the different compartments. FTY720 increases the responsiveness of lymphocytes to homeostatic chemokines, which leads to 
lymphocyte sequestration from circulation to lymph nodes and Peyer's patches, and consequently reduces lymphocyte infiltration to grafts. ${ }^{311} 13$ The chemokine receptor expression itself seems not to be affected by FTY720. ${ }^{13}$

The effect of FTY720 on the distribution of CD4+ T cells in the graft may have a dualistic effect on the rejection process. Reduction of the number of CD4+ helper $\mathrm{T}$ cells will be beneficial because it will lead to a less vigorous graft rejection whereas reduction of the number of CD4+ regulatory T cells will prevent the induction of transplantation tolerance.

After exposure to alloantigens in vivo, alloantigen specific immunoregulatory activity is enriched in a population of CD4+ $\mathrm{T}$ cells that express high levels of CD25. However, the origin, allorecognition properties, and molecular basis for the suppressive activity of CD4+CD25+ regulatory T cells, as well as their relation to other populations of regulatory cells that exist after transplantation, remain a matter of debate. ${ }^{28} 29$ There are no data in the literature regarding the way in which this cell type might be affected by FTY720.

In conclusion, our results show that oral immunosuppression with FTY720 significantly prolongs corneal allograft survival. These results warrant further investigation of FTY720 in preclinical and clinical high risk keratoplasty.

\section{ACKNOWLEDGEMENTS}

The authors thank Novartis for the supply of FTY720 and financial support for animals and reagents.

\section{Authors' affiliations}

K Mayer, F Birnbaum, A Reis, R Sundmacher, Eye Hospital, HeinrichHeine University, Duesseldorf, Germany

T Reinhard, Eye Hospital, Albert-Ludwigs University, Freiburg, Germany S Braunstein, Departement of Pathology, Heinrich-Heine University, Duesseldorf, Germany

F Claas, Department of Immunohematology and Blood Transfusion, Leiden University Medical Center, Leiden, The Netherlands

\section{REFERENCES}

1 Fujita T, Inoue K, Yamamoto S, et al. Fungal metabolites. Part 11. A potent immunosuppressive activity found in Isaria sinclairii metabolite. J Antibiot (Tokyo) 1994;47:208-15.

2 Troncoso $\mathbf{P}$, Stepkowski SM, Wang ME, et al. Prophylaxis of acute renal allograft rejection using FTY720 in combination with subtherapeutic doses of cyclosporine. Transplantation 1999;67:145-51

3 Chiba K, Yanagawa Y, Masubuchi Y, et al. FTY720, a novel immunosuppressant, induces sequestration of circulating mature lymphocytes by acceleration of lymphocyte homing in rats. I. FTY720 selectively decreases the number of circulating mature lymphocytes by acceleration of lymphocyte homing. J Immunol 1998; 160:5037-44.

4 Chiba K, Hoshino Y, Suzuki C, et al. FTY720, a novel immunosuppressant possessing unique mechanisms. I. Prolongation of skin allograft survival and synergistic effect in combination with cyclosporine in rats. Transplant Proc 1996;28:1056-9.

5 Yanagawa Y, Sugahara K, Kataoka H, et al. FTY720, a novel immunosuppressant, induces sequestration of circulating mature lymphocytes by acceleration of lymphocyte homing in rats. II. FTY720 prolongs skin allograft survival by decreasing $T$ cell infiltration into grafts but not cytokine production in vivo. J Immunol 1998; 160:5493-9.
6 Hwang MW, Matsumori A, Furukawa Y, et al. FTY720, a new immunosuppressant, promotes long-term graft survival and inhibits the progression of graft coronary artery disease in a murine model of cardiac transplantation. Circulation 1999;100:1322-9.

7 Kahan BD. FTY720: a new immunosuppressive agent with novel mechanism(s) of action. Transplant Proc 1998;30:2210-13.

8 Ferguson RM, Mulgaonkar S, Tedesco H, et al. FTY720 with reducedexposure Neoral provides adequate rejection prophylaxis in de novo renal transplant recipients. Interim results. XIX International Congress of the Transplantation Society, Miami, FL, USA, 2002.

9 Tedesco H, Kahan B, Mourad G, et al. FTY720 combined with Neoral and Corticosteroids is effective and safe in prevention of acute rejection in renal allograft recipients (interim data). Transplant 2001. Chicago, IL, USA, 2001.

10 Budde K, Schmouder RL, Brunkhorst R, et al. First human trial of FTY720, a novel immunomodulator, in stable renal transplant patients. J Am Soc Nephrol 2002;13:1073-83.

11 Pinschewer DD, Ochsenbein AF, Odermatt B, et al. FTY720 immunosuppression impairs effector $T$ cell peripheral homing without affecting induction, expansion, and memory. J Immunol 2000;164:5761-70.

12 Brinkmann V, Pinschewer D, Chiba K, et al. FTY720: a novel transplantation drug that modulates lymphocyte traffic rather than activation. Trends Pharmacol Sci 2000;21:49-52.

13 Chen S, Bacon KB, Garcia G, et al. FTY720, a novel transplantation drug, modulates lymphocyte migratory responses to chemokines. Transplant Proc 2001;33:3057-63.

14 Brinkmann V, Wilt C, Kristofic C, et al. FTY720: dissection of membrane receptor-operated, stereospecific effects on cell migration from receptorindependent antiproliferative and apoptotic effects. Transplant Proc $2001 ; 33: 3078-80$

15 Mandala S, Hajdu R, Bergstrom J, et al. Alteration of lymphocyte trafficking by sphingosine-1-phosphate receptor agonists. Science 2002;296:346-9.

16 Brinkmann V, Davis MD, Heise CE, et al. The immune modulator FTY720 targets sphingosine 1-phosphate receptors. J Biol Chem 2002;277:21453-7.

17 Herbort CP, Matsubara M, Nishi M, et al. Penetrating keratoplasty in the rat: a model for the study of immunosuppressive treatment of graft rejection. Jpn J Ophthalmol 1989;33:212-20.

18 Reinhard T, Sundmacher R, Godehardt E, et al. Systemische Cyclosporin-AProphylaxe nach Keratoplastiken mit erhöhtem Risiko für Immunreaktionen als einzigem erhöhten Risikofaktor. Ophthalmologe 1997;94:496-500.

19 Reinhard T, Reis A, Bohringer D, et al. Systemic mycophenolate mofetil in comparison with systemic cyclosporin A in high-risk keratoplasty patients: 3 years' results of a randomized prospective clinical trial. Graefe's Arch Clin Exp Ophthalmol 2001;239:367-72.

20 Reis A, Reinhard T, Voiculescu A, et al. Mycophenolate mofetil versus cyclosporin $A$ in high risk keratoplasty patients: a prospectively randomised clinical trial. Br J Ophthalmol 1999;83:1268-71.

21 Hill JC. Systemic cyclosporine in high-risk keratoplasty: long-term results. Eye 1995;9:422-8.

22 Hill JC. The use of cyclosporine in high-risk keratoplasty. Am J Ophthalmology 1989;107:506-10.

23 Vaessen LMB, Mol MM, lizermans JNM, et al. Effects of the novel immunomodulator FTY720 on circulating B cells, NK cells, and T cells expressing the chemokine receptors CCR2, CCR5, CXCR4 and CXCR3 in kidney transplant patients. Am J Transplant 2001;1:165.

24 Bold G, Nickel P, Bitti D, et al. FTY720 induces lymphopenia but does not influence T-lymphocyte and monocyte function in renal transplant patients. Am J Transplant 2002;2:380.

25 Sonoda KH, Taniguchi M, Stein-Streilein J. Long-term survival of cornea allografts is dependent on intact CDId-reactive NKT cells. J Immunol 2002; 168:2028-34.

26 Mayer K, Reinhard T, Reis A, et al. Differential contribution of natural killer cells to corneal graft rejection in 3-week-old versus mature rats. Transplantation 2003;76:578-82.

27 Moser B, Loetscher P. Lymphocyte traffic control by chemokines. Nat Immunol 2001;2:123-8.

28 Wood KJ, Sakaguchi S. Regulatory T cells in transplantation tolerance. Nat Rev Immunol 2003:3:199-210.

29 Francois BJ. Regulatory T cells under scrutiny. Nat Rev Immunol 2003;3:189-98. 